\title{
Analysis On The Association of Intraoperative Fluid Balance and Short-Term Outcomes After Radical Gastrectomy in Aged Patients
}

\section{Yin Cui}

Affiliated Drum Tower Hospital of Medical Department of Nanjing University

\section{Yuhui Wu}

Affiliated Drum Tower Hospital of Medical Department of Nanjing University

\section{Xue Han}

Affiliated Drum Tower Hospital of Medical Department of Nanjing University

Beibei Zhu

Affiliated Drum Tower Hospital of Medical Department of Nanjing University

\section{Zhengliang Ma}

Affiliated Drum Tower Hospital of Medical Department of Nanjing University

Xiaoping Gu ( $\nabla$ xiaopinggu@nju.edu.cn )

Affiliated Drum Tower Hospital of Medical Department of Nanjing University

\section{Research Article}

Keywords: fluid balance, short-term outcomes, radical gastrectomy

Posted Date: April 8th, 2021

DOI: https://doi.org/10.21203/rs.3.rs-342617/v1

License: (c) (1) This work is licensed under a Creative Commons Attribution 4.0 International License. Read Full License 


\section{Abstract}

Background: To observe the relationship between fluid balance and the short-term outcomes of aged patients after gastrectomy for gastric cancer in Nanjing Drum Tower Hospital.

Methods: The clinical data of patients with gastrectomy for gastric cancer from January 2016 to December 2018 in Nanjing Drum Tower Hospital were retrospectively analyzed. According to the criteria of inclusion and exclusion, 691 patients who met the study conditions were analyzed according to intraoperative fluid balance recorded on patients who has undergone radical gastrectomy. Patients were classified into three fluid administration groups representing incremental quartiles of the primary exposure variable. Preoperative characteristics used for statistical adjustment included gender, age, weight, admission type, ASA degree. Operative factors included procedure duration, estimated blood loss, urine output, and so on. The primary outcomes included acute kidney injury (AKI).and postoperative respiratory complications (PRCs) Exploratory outcomes included length of stay, postoperative length of stay and total cost of hospitalization. The association between perioperative factors and AKI/PRCs in hospital was tested with multivariable logistic regression analyses.

Results: 16 cases were diagnosed as AKI and 23 cases were diagnosed as PRCs. The association between intraoperative fluid balance and the incidence of acute kidney injury (AKI)/postoperative respiratory complications (PRCs) remained U-shaped but the difference was not statistically significant $(P>0.05)$. After adjustment for potential confounders, lower urine output $(P=0.017$, $\mathrm{OR}=0.997,95 \% \mathrm{Cl}=0.994-0.999)$ and coronary heart disease $(\mathrm{P}=0.032, \mathrm{OR}=4.867,95 \% \mathrm{Cl}=1.142-20.75)$ were independent predictor of $\mathrm{AKI}$ in aged patients after radical gastrectomy. Besides, coronary heart disease $(\mathrm{OR}=3.371,95 \% \mathrm{Cl}=1.021-11.129, \mathrm{P}=0.049)$ and intestinal obstruction $(\mathrm{OR}=12.501,95 \% \mathrm{Cl}=3.058$ $51.107, \mathrm{P} \otimes 0.0005)$ were independent predictor of PRCs in aged patients after radical gastrectomy.

Conclusion: There were no significant association between the incidence of AKI or any other complications and intraoperative fluid balance during radical gastrectomy in aged patients. Lower urine output and coronary heart disease were independent predictors of AKI in aged patients after radical gastrectomy. Coronary heart disease and intestinal obstruction were independent predictors of PRCs in aged patients after radical gastrectomy.

Trial registration: This study was approved by the Affiliated Drum Tower Hospital of Nanjing University (Registration number: 2018-162-01).

\section{Background}

At least 310 million operations took place each year and this number is still rising nowadays [1]. Perioperative fluid management, one of the key components of enhanced recovery pathways, has been widely used in surgical procedures. Optimal intraoperative fluid management is important because both under and over fluid balance (FB) may associated with harm [2-5]. Prior studies found that exposure to positive or negative FB was associated with long-term mortality compared with even FB [6], while another 
trial recently found that even FB was associated with a higher rate of acute kidney injury [7]. Therefore, the evidence for fluid therapy during and immediately after surgery is still inconclusive.

Fluid therapy should only be given in well-defined protocols according to individual needs [8, 9], especially the aged [10]. Markedly increased cases of operations in fast-elderly population have posed a significant challenge to medical health field. Elderly subjects have reduced ability of regulating homeostasis, decreased myocardial function and impaired pulmonary reserve function, and these may significantly increase susceptibility for multiple diseases after surgery. Thus, identifying an optimal strategy of fluid resuscitation for elderly patients during perioperative will improve outcomes.

Therefore, aged patients undergone gastric cancer radical surgery were chosen, a common surgical operation. We conducted this retrospective study to explore the optimal strategy of fluid therapy on postoperative clinical outcomes like acute renal injury (AKI), postoperative respiratory complications (PRCs) and so on. We also tried to identify the independent predictors of such complications.

\section{Methods}

\section{Participants}

All methods were carried out in accordance with relevant guidelines and regulations of the Affiliated Drum Tower Hospital of Nanjing University. Since it is not harmful to patients with data collected, a statement on consent waiver for this study was approved by Ethics Committee of the Affiliated Drum Tower Hospital of Nanjing University (Registration number: 2018-162-01). Subjects in this research were consecutively recruited from January 2016 to December 2018 and undergoing gastric cancer radical surgery. The

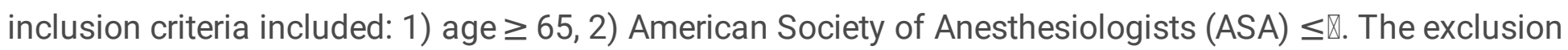
criteria were as follows: 1) no renal function test was performed during the perioperative period. 2) hepatic and or kidney dysfunction. 3) vital cardiovascular dysfunction 4) preoperative coagulation dysfunction 5) severe surgery within the past year 6) incomplete medical records. A total of 691 subjects were enrolled. The subjects were divided into 3 groups according to the intraoperative liquid intake (input minus output) by percentile method: low intake group (<25\%), medium intake group $(26 \% \sim 75 \%)$, and high intake group (> 75\%). The subjects were further divided into AKI group and non-AKI group according to whether acute renal injury (AKI) developed or not. The subjects were divided into PRCs groups and nonPRCs according to whether postoperative respiratory complications (PRCs) appeared.

Anesthesia treatment

All participants received general anesthesia according to a standardized protocol. All patients received standard monitoring, heart rate, arterial pressure, central venous pressure, PETCO2, SpO2 and body temperature were recorded continuously. All aspects of clinical care were documented in each patient's medical record.

Short-term prognosis after operation 
All patients were transferred to PACU or ICU after operation and experienced the process of recovery. They were transferred to the general ward when condition stable. The time of defecation was recorded to judge the recovery of intestinal function. Urine volume and serum creatinine were recorded to judge whether acute renal injury (AKI) developed or not after surgery [11]. In this study, PRCs were recorded with the definition of pneumonia or pulmonary edema with imaging evidence, respiratory failure, and / or intubation within 3 days after surgery.

Statistical analysis

The measurement data which presented as the mean \pm SD or Median (Quartiles) were statistically analyzed with One-way ANOVA, Kruakal Wallis rank sum test or $t$ test. The numeration data were statistically analyzed with Fisher test or $\chi 2$ test. All statistics were performed with SPSS v24.0, Empowerstats software and $\mathrm{R}$ software. $\mathrm{P}<0.05$ was considered to be statistically significant.

\section{Results}

\section{Prediction of AKI occurrence}

In this study, 16 patients fulfilled the diagnostic criteria for AKI. The incidence rate of AKI was $2.32 \%$ (16/691) during hospitalization. The patients of AKI group were older ( 74.5 vs $71.85, P=0.036)$, with higher anemia and coronary disease incidence ( $62.5 \%$ vs $36.89 \%, P=0.036 ; 18.75 \%$ vs $4.15 \%, P=0.031$ ) and lower albumin level preoperative (36.54 vs 38.32, $\mathrm{P}=0.028)$. The use of Dizosin was lower in AKI group when compared with non-AKI group intraoperative (43.75\% vs $69.19 \%, P=0.03)$. The AKI group has a higher in-hospital mortality $(6.25 \%$ vs $0.3 \%, P=0.068)$, postoperative length of stay $(16$ vs $11, P<$ $0.001)$, total length of stay ( 26 vs $18, P<0.001)$ and total cost of hospitalization (131737.29 vs 75083.1 , $\mathrm{P}<0.001)$. Table.1.

After adjusting for confounding variables (age, albumin level preoperative, anemia, use of Dizosin and liquid balance), Preoperative coronary disease (OR 4.867, 95\% Cl: 1.142-20.750, $\mathrm{P}=0.032$ )and decreased urine output (OR $0.997,95 \% \mathrm{Cl}: 0.994-0.999, \mathrm{P}=0.017$ ) were respectively independent risk factors for the incidence of AKI in elderly patients after radical gastrectomy (Table 2). After put the intraoperative net fluid intake and intraoperative fluid balance group as exposure variables and adjusted for confounding variables like age, albumin level preoperative, preoperative coronary disease and so on, no correlation has been found between the net fluid input and the incidence of AKI in elderly patients after radical gastrectomy (Table 3).

\section{Prediction of PRCs occurrence}

Among these 691 patients, PRCs was detected in 23 (3.33\%) during hospitalization. Compared with nonPRCs group, $P R C s$ patients have an older age (74.48 vs $71.82, P=0.01)$, increased hypertension $(65.22 \%$ vs $44.10 \%, P=0.044)$, coronary disease $(17.39 \%$ vs $4.05 \%, P=0.016)$ and digestive tract obstruction incidence $(13.04 \%$ vs $1.95 \%, P=0.014)$. The intraoperative urine volume of PRCs group was lower than 
that of non PRCs group (300 vs 500, P = 0.03). The postoperative hospitalization time and total hospitalization time were significantly longer (15.43 vs $12.95, P=0.003 ; 22.7$ vs 19.07$)$. The total cost of hospitalization increased markedly (94333.17 vs 75779.37, $\mathrm{P}<0.001$ ). (Table 4)

After adjusting for confounding variables (age, hypertension and so on), the logistic regression analysis identified that the occurrence of coronary disease (OR 3.371, 95\% Cl: $1.021-11.129, \mathrm{P}=0.049)$ and digestive tract obstruction before surgery(OR 12.501, 95\% Cl: 3.058-51.107, $\mathrm{P}<0.0005)$ remained independent predictors of the incidence of PRCs in elderly patients after radical gastrectomy. (Table 5A). Put the intraoperative net liquid intake and intraoperative fluid balance group as exposure variables and adjusted for confounding variables like age, hypertension, preoperative coronary disease and so on, no links has been shown between the net fluid input and the incidence of PRCs in elderly patients after radical gastrectomy (Table 5B).

\section{Prediction of defecating time}

The average defecation time was $5.79 \pm 2.25$ days in these 691 patients included. the univariate analysis showed that colloidal infusion volume, crystal and colloid ratio, baseline C-reactive protein level, hypoalbuminemia and use of vasoactive drugs during operation were suspected factors of influencing postoperative defecation time (Table 6A). After adjusting for these suspected variables, we found no relationship between the net fluid input and defecating time in elderly patients after radical gastrectomy (Table 6B).

\section{Discussion}

Fluid therapy serves to achieve homeostasis by restoring and maintaining body water, electrolytes and tissue perfusion $[12,13]$. ERAS programs recommended to avoid too much intravenous fluid $[14,15]$. Some studies determined restrictive fluid therapy to be optimal which reduced the complication rate compared with liberal fluid management policy [16]. However, inappropriate fluid-balance approaches may be harmful $[2,17]$. Our findings showed that the incidence of AKI and PRCs in low intake group and high intake group increased when compared with medium intake group, no significant difference although. That is, liquid balance is not associated with postoperative clinical outcomes in aged patients undergone gastric cancer radical surgery. Myles' research found that restrictive fluid regimen was not associated with increased rate of disability-free survival and was associated with increased incidence of AKI compared with a liberal fluid therapy [7]. We suggest that the differences in the observed results may be due to our small sample size relatively and a single-center study.

AKI occurrence after operation could induce increased mortality, hospitalization and medical expenses [18-19]. In our trial the mortality of AKI group was $6.25 \%$ while non-AKI group was $0.3 \%$ in hospital after operation $(P=0.068)$. No significant difference was found which may due to short follow-up time. However, noted that one third of the dead cases have been suffered from AKI. In addition, AKI group has longer hospital stays. Some studies found that body mass index, hyperlipidemia, preoperative use of ACE-I or ARB, COPD and diabetes could be the independent risk factors for AKI after postoperative [20- 
23]. Our findings showed that aged, anemia, preoperative coronary disease incidence low albumin level preoperative, decreased urine output and the use of Dizosin were associated with increased frequency of AKI. By multivariable analyses, the independent risk factors for AKI were preoperative coronary disease and decreased urine output. The decrease of urine volume during operation is the external sign of renal perfusion insufficiency, which is related to the occurrence of AKI. While in this study, we did not find that COPD, diabetes and so on were AKI Independent risk factors. We thought that the event rate of AKI was relatively low, which may have limited our ability to test for a large number of risk factors in a multivariable model.

Our study analyzed the risk for PRCs that is linked with commonly prescribed medications for comorbid conditions in subjects who undergo radical gastrectomy. The data indicate the occurrence of coronary disease and digestive tract obstruction preoperative were associated with increased odds for development of PRCs. Interestingly, Coronary heart disease preoperative turned out to be an independent risk factor both of AKI and PRCs in elderly patients after radical gastrectomy. The possible mechanism is that patients with a long history of coronary heart disease may result in ischemic cardiomyopathy, decreased cardiac contractility, and reduced cardiac output. The decrease of renal blood flow, difficult of pulmonary venous return, increased pressure in pulmonary capillaries and pulmonary interstitial edema, and this eventually developed into AKI and PRCs. Patients with digestive tract obstruction before operation are of inflammation and water electrolyte disorder in degree, which may be a potential mechanism of PRCs.

\section{Conclusion}

In conclusion, in patients at aged for complications while undergoing radical gastrectomy, fluid therapy was not associated with a higher rate of AKI and PRCs. Preoperative coronary disease and decreased urine output are independently associated with increased risk for postoperative AKI. Coronary disease and digestive tract obstruction preoperative were associated with increased incidence of PRCs. Limited by its retrospective design and relatively small sample size in our study, we suggest a larger-scale prospective study be conducted in future.

\section{Abbreviations}

AKI: acute kidney injury

PRCs: postoperative respiratory complications

FB: fluid balance

ASA: American Society of Anesthesiologists

\section{Declarations}


Ethics approval and consent to participate: This study was approved by the Affiliated Drum Tower Hospital of Nanjing University (Registration number: 2018-162-01).

Consent for publication: Not applicable.

Availability of data and materials: We declared that materials described in the manuscript, including all relevant raw data, will be freely available to any scientist wishing to use them for non-commercial purposes, without breaching participant confidentiality. If anyone wants to request the data from this study, please contact with corresponding author.

Competing interests: The authors declare that they have no competing interests.

Funding: This research was supported by National Natural Science Foundation of China (81730033) and the Key Talent's of 13th Five-Year Plan for Strengthening Health of Jiangsu Province『ZDRCA2016069『

Authors' contributions: Yin Cui has seen the original study data, reviewed the analysis of the data, and was a major contributor in writing the manuscript. Yuhui Wu designed the study, conduct the study, analyze the data. Xue Han conducted the study. Beibei Zhu analyze the data. Zhengliang Ma helped design the study and write the manuscript. Xiaoping Gu helped design the study and write the manuscript. All authors read and approved the final manuscript.

Acknowledgements: Not applicable.

\section{References}

1 Weiser T G, Haynes A B, Molina G, et al. Estimate of the global volume of surgery in 2012: an assessment supporting improved health outcomes[J]. The Lancet, 2015, 385: S11.

2 BELLAMY M C. Wet, dry or something else?[J]. British journal of anaesthesia, 2006, 97(6): 755-757.

3 Miller T E, Myles P S. Perioperative fluid therapy for major surgery[J]. Anesthesiology: The Journal of the American Society of Anesthesiologists, 2019, 130(5): 825-832.

4 Martínez-García J J, León-Sicairos N M, Canizalez-Roman A, et al. Fluid balance and acute kidney injury in septic shock[J]. Boletín Médico Del Hospital Infantil de México (English Edition), 2017, 74(4): 282-288.

5 Ratner A J, Lysenko E S, Paul M N, et al. Synergistic proinflammatory responses induced by polymicrobial colonization of epithelial surfaces[J]. Proceedings of the National Academy of Sciences, 2005, 102(9): 3429-3434.

6 Balakumar V, Murugan R, Sileanu F E, et al. Both positive and negative fluid balance may be associated with reduced long-term survival in the critically ill[J]. Critical care medicine, 2017, 45(8): e749. 
7 Myles P S, Bellomo R, Corcoran T, et al. Restrictive versus liberal fluid therapy for major abdominal surgery[J]. New England Journal of Medicine, 2018, 378(24): 2263-2274.

8 Myles P S, Andrews S, Nicholson J, et al. Contemporary approaches to perioperative IV fluid therapy[J]. World journal of surgery, 2017, 41(10): 2457-2463.

9 Jin J, Min S, Liu D, et al. Clinical and economic impact of goal-directed fluid therapy during elective gastrointestinal surgery[J]. Perioperative Medicine, 2018, 7(1): 22.

10 Lou X, Lu G, Zhao M, et al. Preoperative fluid management in traumatic shock: a retrospective study for identifying optimal therapy of fluid resuscitation for aged patients[J]. Medicine, 2018, 97(8).

11 Levey A S, De Jong P E, Coresh J, et al. The definition, classification, and prognosis of chronic kidney disease: a KDIGO Controversies Conference report[J]. Kidney international, 2011, 80(1): 17-28.

12 Feldheiser A, Aziz O, Baldini G, et al. Enhanced Recovery After Surgery (ERAS) for gastrointestinal surgery, part 2: consensus statement for anaesthesia practice[J]. Acta Anaesthesiologica Scandinavica, 2016, 60(3): 289-334.

13 Varadhan K K, Lobo D N. A meta-analysis of randomised controlled trials of intravenous fluid therapy in major elective open abdominal surgery: getting the balance right[J]. Proceedings of the Nutrition Society, 2010, 69(4): 488-498.

14 Gustafsson U O, Scott M J, Schwenk W, et al. Guidelines for perioperative care in elective colonic surgery: Enhanced Recovery After Surgery (ERAS $\AA$ ) Society recommendations[J]. World journal of surgery, 2013, 37(2): 259-284.

15 Bundgaard-Nielsen $\mathrm{M}$, Secher $\mathrm{N} \mathrm{H}$, Kehlet $\mathrm{H}$. 'Liberal' vs 'restrictive' perioperative fluid therapy-a critical assessment of the evidence[J]. Acta Anaesthesiologica Scandinavica, 2009, 53(7): 843-851.

16 Schol P B B, Terink I M, Lance M D, et al. Liberal or restrictive fluid management during elective surgery: a systematic review and meta-analysis[J]. Journal of clinical anesthesia, 2016, 35: 26-39.

17 Thacker J K M, Mountford W K, Ernst F R, et al. Perioperative fluid utilization variability and association with outcomes[J]. Annals of surgery, 2016, 263(3): 502-510

18 Obermüller N, Geiger H, Weipert C, et al. Current developments in early diagnosis of acute kidney injury[J]. International urology and nephrology, 2014, 46(1): 1-7.

19 Lafrance J P, Miller D R. Acute kidney injury associates with increased long-term mortality[J]. Journal of the American Society of Nephrology, 2010, 21(2): 345-352.

20 Lombardi R, Ferreiro A. Risk factors profile for acute kidney injury after cardiac surgery is different according to the level of baseline renal function[J]. Renal failure, 2008, 30(2): 155-160. 
21 Arora P, Rajagopalam S, Ranjan R, et al. Preoperative use of angiotensin-converting enzyme inhibitors/angiotensin receptor blockers is associated with increased risk for acute kidney injury after cardiovascular surgery[J]. Clinical Journal of the American Society of Nephrology, 2008, 3(5): 1266-1273.

22 Karkouti K, Wijeysundera D N, Yau T M, et al. Acute kidney injury after cardiac surgery[J]. Circulation, 2009, 119(4): 495-502.

23 Thakar C V, Kharat V, Blanck S, et al. Acute kidney injury after gastric bypass surgery[J]. Clinical Journal of the American Society of Nephrology, 2007, 2(3): 426-430.

\section{Tables}

Table1 Comparison of perioperative factors between AKI group and non-AKI group. [n (\%), $\pm \mathrm{s}, \mathrm{M}(\mathrm{Q} 1, \mathrm{Q} 3)]$ 


\begin{tabular}{|c|c|c|c|}
\hline factors & $\mathrm{AKI}$ & non-AKI & $P$ value \\
\hline cases & 16 & 675 & \\
\hline \multicolumn{4}{|l|}{ preoperatige } \\
\hline male $(x)$ & $15(93.75)$ & $548(81.19)$ & 0.784 \\
\hline age (year) & $74.50 \pm 5.23$ & $71.85 \pm 4.88$ & 0.036 \\
\hline ASA degree $(x)$ & & & 0.137 \\
\hline II & $1(6.25)$ & $169(25.04)$ & \\
\hline III & $15(93.75)$ & $506(74.96)$ & \\
\hline anem ia $(0)$ & $10(62.50)$ & $249(36.89)$ & 0.036 \\
\hline hypoalbum inem ia (\%) & $0(0,00)$ & $5(0.74)$ & 1.000 \\
\hline Hypertension $(x)$ & $10(62.50)$ & $299(44.30)$ & 0.203 \\
\hline D iabetes $(x)$ & $0(0.00)$ & $86(12.74)$ & 0.243 \\
\hline coronary disease $(x)$ & $3(18.75)$ & $28(4.15)$ & 0.031 \\
\hline Periphera1 vascular disease (x) & $0(0.00)$ & $2(0.30)$ & 1.000 \\
\hline history of cerebral infarction $(x)$ & $1(6.25)$ & $61(9.05)$ & 1.000 \\
\hline sm oking history $(x)$ & $2(12.50)$ & $93(13.80)$ & 1.000 \\
\hline chronic bronchitis(x) & $0(0.00)$ & $7(1.05)$ & 1.000 \\
\hline em physem a $(x)$ & $2(12.50)$ & $16(2.37)$ & 0.062 \\
\hline $\operatorname{COPD}\left(W_{0}\right)$ & $0(0.00)$ & $6(0.89)$ & 1.000 \\
\hline \multicolumn{4}{|l|}{ preopera tive gastro in testinal } \\
\hline obstruction $(\%)$ & $1(6.25)$ & $15(2.22)$ & 0.315 \\
\hline weight(kg) & $63.67 \pm 12.45$ & $63.26 \pm 11.26$ & 0.691 \\
\hline baseline crea tinine (um o1/1) & $71.06 \pm 15.15$ & $69.45 \pm 13.23$ & 0.687 \\
\hline Baseline to talpro tein $(\mathrm{g} / \mathrm{L})$ & $59.44 \pm 6.34$ & $61.02 \pm 5.83$ & 0.421 \\
\hline Baseline atbum in $(g / L)$ & $36.54 \pm 3.09$ & $38.32 \pm 3.21$ & 0.028 \\
\hline Baseline $\mathrm{C}$-reactive pro tein (d) & $4.70(2.85,5.80)$ & $3.50(2.50,5.80)$ & 0.639 \\
\hline baseline eG FR (m $1 / \mathrm{m}$ in $/ 1.73 \mathrm{~m}^{2}$ ) & $101.89 \pm 21.14$ & $102.23 \pm 20.74$ & 0.972 \\
\hline baseline fasting $\mathrm{b}$ lood & & & \\
\hline sugar(FBS) (m mo1/L) & $5.16 \pm 1.83$ & $5.12 \pm 1.17$ & 0.738 \\
\hline \multicolumn{4}{|l|}{ operative } \\
\hline operative duration (h) & $4.04 \pm 0.86$ & $3.90 \pm 1.07$ & 0.352 \\
\hline Type $\left(W_{0}\right)$ & & & 0.616 \\
\hline laparoscope & $0(1.00)$ & $43(6.37)$ & \\
\hline laparotom y & $16(100.00)$ & $632(93.63)$ & \\
\hline Furosem ide $(x)$ & $3(18.75)$ & $87(12.89)$ & 0.452 \\
\hline $\mathrm{U}$ linasta tin (w) & $5(31.25)$ & $204(30.22)$ & 1.000 \\
\hline D exam ethasone $(x)$ & $5(31.25)$ & $245(36.30)$ & 0.678 \\
\hline Dezocine $(x)$ & $7(43.75)$ & $467(69.19)$ & 0.030 \\
\hline sevo fhurane $(x)$ & $5(31.25)$ & $107(15.85)$ & 0.158 \\
\hline norm a1 saline (m 1) & $0.00(0.00,25.00)$ & $0.00(0.00,0.00)$ & 0.203 \\
\hline Compound sodium chloride (m 1 ) & $0.00(0.00,1000.00)$ & $0.00(0.00,500.00)$ & 0.997 \\
\hline \multicolumn{4}{|c|}{ Sodium lacta te Ringer } \\
\hline solution (m 1$)$ & $843.75 \pm 625.00$ & $790.59 \pm 483.4$ & 0.947 \\
\hline crysta1(m 1) & $1425.00 \pm 502.66$ & $1505.73 \pm 465.41$ & 0.720 \\
\hline Succinylgelatin (m 1) & $0.00(0.00,500.00)$ & $0.00(0.00,500.00)$ & 0.996 \\
\hline Hydroxyethy1starch (m 1$)$ & $637.50 \pm 464.58$ & $766.67 \pm 505.49$ & 0.251 \\
\hline collo id (m 1$)$ & $887.50 . \pm 330.40$ & $1034.59 \pm 326.24$ & 0.056 \\
\hline colloid types(x) & & & 0.983 \\
\hline no useage of clloid & $0(0.00)$ & $2(0.30)$ & \\
\hline Hydroxye thyl starch & $11(68.75)$ & $469(69.48)$ & \\
\hline Succinylgelatin & $4(25.00)$ & $150(22.22)$ & \\
\hline both & $1(6.25)$ & $54(8.00)$ & \\
\hline Crysta1 colloid ratio & $1.70 \pm 0.59$ & $1.57 \pm 0.68$ & 0.224 \\
\hline b lood transfusion (m 1) & $0.00(0.00,1000.00)$ & $0.00(0.00,0.00)$ & 0.558 \\
\hline blood loss(m 1) & $303.12 \pm 222.46$ & $237.29 \pm 197.46$ & 0.114 \\
\hline Urine vo lum e (m 1) & $275.00(200.00,425.00)$ & $500.00(300.00,800.00)$ & 0.002 \\
\hline to ta1 input (m 1) & $2318.75 \pm 735.05$ & $2546.47 \pm 650.39$ & 0.324 \\
\hline to toloutput (m 1) & $700.00 \pm 360.56$ & $877.76 \pm 521.00$ & 0.168 \\
\hline inputrela tively (m 1$)$ & $1618.75 \pm 567.71$ & $1668.71 \pm 563.37$ & 0.881 \\
\hline \multicolumn{4}{|l|}{ inputrela tively divided in to } \\
\hline groups( $)$ & & & 0.244 \\
\hline low & $6(37.50)$ & $146(21.63)$ & \\
\hline m edium & $5(31.25)$ & $326(48.30)$ & \\
\hline high & $5(31.25)$ & $203(30.07)$ & \\
\hline \multicolumn{4}{|l|}{ postoperative } \\
\hline to ta 1 cost(yuan) & $131737.29 \pm 57215.91$ & $75083.10 \pm 15489.76$ & $<0.001$ \\
\hline postopera tive hosp ita1stay (d) & $16(14,27)$ & $11(10,14)$ & $<0.001$ \\
\hline to ta1stay $(d)$ & $26(19,31)$ & $18(15,21)$ & $<0.001$ \\
\hline postoperative defecation tim e(d) & $4(3.5,8.5)$ & $6(4,7)$ & 0.633 \\
\hline anastom tic leakage( & $1(6.25)$ & $8(1.19)$ & 0.191 \\
\hline a second operation $(x)$ & $2(12.50)$ & $5(0.74)$ & 0.010 \\
\hline in-hosp ita1 death $(x)$ & $1(6.25)$ & $2(0.30)$ & 0.068 \\
\hline pulm onary com plications $(\%)$ & $3(18.75)$ & $20(2.96)$ & 0.014 \\
\hline
\end{tabular}

Table2 Regression model analysis of risk factors for the incidence of AKI 


\begin{tabular}{|c|c|c|c|c|}
\hline $\begin{array}{l}\text { Predictors } \\
\text { age (year) }\end{array}$ & $\begin{array}{l}\text { OR } 95 \% \mathrm{CD} \\
1.1(1.0,1.2)\end{array}$ & $\begin{array}{l}P \text { value } \\
\text { D_o36 }\end{array}$ & $\begin{array}{l}O R \% 5 \% C D \\
1.051(0.953,1.159)\end{array}$ & $\begin{array}{l}P \text { value } \\
0317\end{array}$ \\
\hline Baseline to ta 1 & & & & \\
\hline protein $(g / L)$ & $0.8(0.7,1,0)$ & 0.029 & $0.866(0.713,1105)$ & 0_144 \\
\hline anem ia & $29(1,0,79)$ & 0_045 & $1364(0399,4$,668) & 0.621 \\
\hline coronary disease & $53(1.4,19.8)$ & 0_012 & $4.867(1.142,20.75)$ & 0_032 \\
\hline Dezocine & $03(0,1,09)$ & 0_038 & $0357\left(0.125,1 \_022\right)$ & 0.055 \\
\hline colloid vohum e (m 1$)$ & 1_0 $\left(1 \_0,1 \_0\right)$ & 0_or2 & 1_0（0998,1_113) & 0.692 \\
\hline $\begin{array}{l}\text { Urine volum e }(\mathrm{m} 1) \\
\text { inputrelatively divided } \\
\text { in to groups }\end{array}$ & 1_0 $\left(1 \_0,1 \_0\right)$ & 0_013 & $0997(0994,0999)$ & 0.017 \\
\hline low & control & & confrol & \\
\hline m edium & $0.4(0,1,12)$ & 0_108 & $0.577(0.206,1.615)$ & 0.152 \\
\hline high & $0.6(02,20)$ & 0_405 & $0.617(0.195,1953)$ & 0_480 \\
\hline
\end{tabular}

Table 3 Relationship between intraoperative net fluid input and the incidence of AKI postoperative in different models

uncorrected

\begin{tabular}{|c|c|c|c|c|}
\hline exposure variables & OR $95 \% C D$ & $P$ value & OR $(95 \% C D$ & $P$ value \\
\hline \multicolumn{5}{|l|}{ input } \\
\hline rela tively $(100 \mathrm{~m} 1)$ & $0985(0902,1.074)$ & 0.726 & $1 \_016(0.896,1.153)$ & 0.800 \\
\hline \multicolumn{5}{|l|}{ inputrelatively } \\
\hline \multicolumn{5}{|l|}{ divided into groups } \\
\hline low & control & & control & \\
\hline m edium & $0.373(0.112,1245)$ & 0_108 & $0.329(0.072,1506)$ & 0.152 \\
\hline high & $0.599(0.18,2001)$ & $0 \_405$ & $0.517(0.084,3202)$ & 0.480 \\
\hline$P$ for trend & & 0.420 & & 0.604 \\
\hline
\end{tabular}

Table4 Comparison of perioperative factors between PRCs group and non-PRCs group. [n (\%), \pm $s, \mathrm{M}(\mathrm{Q} 1, \mathrm{Q} 3)]$ 


\begin{tabular}{|c|c|c|c|}
\hline factors & PRCs & non-PRCs & $P$ value \\
\hline cases & 23 & 668 & \\
\hline \multicolumn{4}{|l|}{ preoperative } \\
\hline $\mathrm{m}$ ale $(\mathrm{w})$ & $20(8696)$ & $543(8129)$ & 0.784 \\
\hline age (year) & $74.48 \pm 4.97$ & $71.82 \pm 4.88$ & 0.010 \\
\hline ASA degree $(\%)$ & & & 0.137 \\
\hline II & $6(26.09)$ & $164(24.55)$ & \\
\hline III & $17(7391)$ & $504(75-45)$ & \\
\hline anem ia $(x)$ & $11(47.83)$ & $248(37.13)$ & 0381 \\
\hline hypoatbum inem ia (⿻) & $0(0,00)$ & $5(0.75)$ & 1.000 \\
\hline Hypertension $(y)$ & $15(6522)$ & $294(44.01)$ & 0.044 \\
\hline D iabetes $(x)$ & $2(8.70)$ & $84(1257)$ & 0.757 \\
\hline coronary disease $(x)$ & $4(1739)$ & $27(4.05)$ & 0.016 \\
\hline Periphera1vascular disease $(*)$ & $0(0.00)$ & $2(0.30)$ & 1.000 \\
\hline history of cerebral in farction $\left(\psi_{i}\right)$ & $0(0.00)$ & $62(930)$ & 0254 \\
\hline sm oking history $(x)$ & $3\left(13 \_04\right)$ & $92(13.79)$ & 1.000 \\
\hline chronic bronchitis (W) & $0(0.00)$ & $7(1.05)$ & 1.000 \\
\hline em physem a $(x)$ & $2(8.70)$ & $16(240)$ & 0.117 \\
\hline $\operatorname{COPD}\left(w_{1}\right)$ & $0(0.00)$ & $6(090)$ & 1.000 \\
\hline \multicolumn{4}{|l|}{ preopera tive gastro in testina 1} \\
\hline obstruction $(x)$ & $3(13.04)$ & 13 (195) & 0.014 \\
\hline weight(Kg) & $61.45 \pm 8.97$ & $6334 \pm 1135$ & 0.425 \\
\hline baseline crea tinine (um o1/1) & $68.26 \pm 1293$ & $6953 \pm 1328$ & 0.669 \\
\hline Baseline to ta1protein $(g / L)$ & $60.35 \pm 736$ & $61.01 \pm 5.79$ & 0.899 \\
\hline Baseline album in $(g / L)$ & $38 \pm 339$ & $3828 \pm 321$ & 0.643 \\
\hline Baseline C-reactive pro tein (d) & $430(280,10.05)$ & $350(250,5.75)$ & 0.178 \\
\hline baseline eG FR $\left(\mathrm{m} 1 / \mathrm{m}\right.$ in $/ 1.73 \mathrm{~m}^{2}$ ) & $104.67 \pm 2130$ & $102.14 \pm 20.73$ & 0.663 \\
\hline \multicolumn{4}{|c|}{ baseline fasting $b$ lood } \\
\hline sugar(FBS) (m mo1/L) & $498 \pm 125$ & $5.13 \pm 1.18$ & 0.328 \\
\hline \multicolumn{4}{|l|}{ operative } \\
\hline operative duration $(h)$ & $4.07 \pm 0.97$ & $3.90 \pm 1.07$ & 0327 \\
\hline Type (y) & & & 0389 \\
\hline laparoscope & $0(0.00)$ & $43(6.44)$ & \\
\hline laparo tom y & $23(100.00)$ & $625(9356)$ & \\
\hline Furosem ide $(\%)$ & $2(8.70)$ & $88(13.17)$ & 0.756 \\
\hline $\mathrm{U}$ linastatin $(x)$ & $7(30.43)$ & $202(30.24)$ & 0.984 \\
\hline D exam ethasone $(\mathbb{y})$ & $7(30.43)$ & $243(3638)$ & 0.662 \\
\hline Dezocine $(x)$ & $17(7391)$ & $457(68.41)$ & 0.655 \\
\hline sevofhurane $(x)$ & $3(13.04)$ & $109(1632)$ & 1000 \\
\hline nom al saline (m 1$)$ & $0(0,0)$ & $0(0,0)$ & 0.191 \\
\hline Com pound sodium chloride (m 1 ) & $0(0,500)$ & $0(0,500)$ & 0.535 \\
\hline \multicolumn{4}{|c|}{ Sodium lacta te Ringer } \\
\hline solution (m 1) & $747.83 \pm 461.10$ & $79334 \pm 487.75$ & 0.679 \\
\hline crysta1(m 1) & $146957 \pm 438.42$ & $1505 \_04 \pm 46726$ & 0.548 \\
\hline Succiny1gelatin (m 1$)$ & $0(0,500)$ & $0(0,500)$ & 0.978 \\
\hline Hydroxyethylstarch (m 1$)$ & $782.61 \pm 560.56$ & $763.02+503 \_06$ & 0.743 \\
\hline collo id (m 1) & $1043 \_48 \pm 366599$ & $1030.76 \pm 325.69$ & 0.746 \\
\hline colloid types $(x)$ & & & 0.893 \\
\hline no useage of clloid & $0(0.00)$ & $2(0.30)$ & \\
\hline Hydroxye thylstarch & $16(6957)$ & $464(69-46)$ & \\
\hline Succinylgelatin & $6(26.09)$ & $148(2216)$ & \\
\hline both & $1(4.35)$ & 54 (8.08) & \\
\hline Crysta1 colloid ratio & $158+0.69$ & 1570.68 & 0.770 \\
\hline blood transfusion (m 1) & 0 $(0,0)$ & $0(0,0)$ & 0.759 \\
\hline blood loss(m 1) & $280.43 \pm 15936$ & $23738 \pm 19928$ & 0.083 \\
\hline Urine vo hum e (m 1) & $300(250,550)$ & $500(300,800)$ & 0.043 \\
\hline to ta1 input (m 1) & $2513.04 \pm 67238$ & 2542.1765259 & 0.671 \\
\hline to toloutput (m 1) & $893 \_48 \pm 755 \_18$ & $87296+509.05$ & 0.292 \\
\hline inputrelatively (m 1) & $161957 \pm 736.04$ & $166921 \pm 556.85$ & 0.661 \\
\hline \multicolumn{4}{|l|}{ inputrela tively divided in to } \\
\hline groups(x) & & $144(2150)$ & 0329 \\
\hline low & $8(34.78)$ & & \\
\hline m edium & $9(39.13)$ & $322(48.20)$ & \\
\hline high & $6(26.09)$ & $202(30.24)$ & \\
\hline \multicolumn{4}{|l|}{ postoperative } \\
\hline to ta 1 cost(yuan) & $94333.17 \pm 2453790$ & $7577937 \pm 18981.69$ & $<0.001$ \\
\hline postopera tive hosp ita1stay (d) & $15.43 \pm 755$ & $12.95 \pm 6.48$ & 0.003 \\
\hline to ta1stay (d) & $2270 \pm 8.40$ & $19.07 \pm 7.10$ & $<0.001$ \\
\hline postoperative defecation tim e (d) & $638 \pm 278$ & $5.72 \pm 2.27$ & 0.193 \\
\hline anastom tic leakage (W) & $1(4.35)$ & $8(120)$ & 0.264 \\
\hline a second operation (w) & $1(4.35)$ & $6(090)$ & 0.212 \\
\hline in-hosp ita1death $(x)$ & $0(0.00)$ & $3(0.45)$ & 1.000 \\
\hline
\end{tabular}

Table5A Regression model analysis of risk factors for the incidence of PRCs 


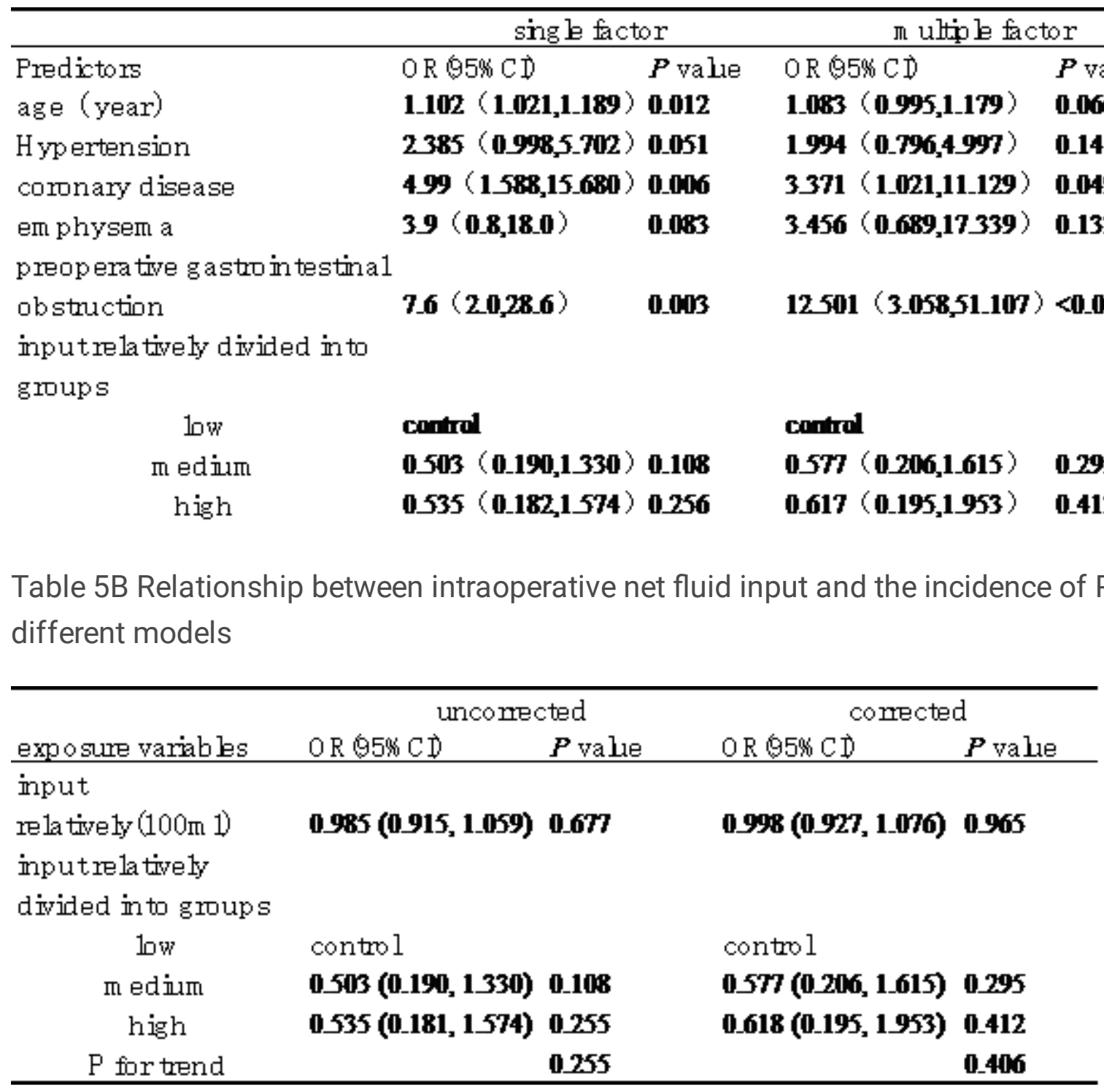

Table 6A Single factor analysis of postoperative defecation time

\begin{tabular}{|c|c|c|c|}
\hline \multirow[b]{2}{*}{ Predictors } & \multirow[b]{2}{*}{$\beta$} & \multicolumn{2}{|c|}{ single factor } \\
\hline & & $95 \% \mathrm{CI}$ & $P$ value \\
\hline \multicolumn{4}{|l|}{ Baseline $\mathrm{C}$-reactive } \\
\hline protein & D_t109 & $-0.013-0.021$ & 0.128 \\
\hline hypoatbum inem ia & 23 & 03-43 & 0.027 \\
\hline use of vasoactive drugs & -1 & $-1.7-02$ & 0_to6 \\
\hline colloid & 0_001 & -0_0101021-0_001 & 0.1600 \\
\hline Crystalcolloid ratio & -0.237 & -0_493-0_019 & 0.070 \\
\hline
\end{tabular}

Table 6B The relationship between the net fluid input and defecating time after radical gastrectomy 


\begin{tabular}{|c|c|c|c|}
\hline \multirow[b]{2}{*}{ exposure variables } & uncomected & \multicolumn{2}{|l|}{ comected } \\
\hline & B95\% CD & B95\% CD & $P$ value \\
\hline \multicolumn{4}{|l|}{ input } \\
\hline rela tively $(100 \mathrm{~m} 1)$ & 0.015 (-0_026, 0_036) 0.7526 & -0_0III2 (-0_039,0_038) & 0991 \\
\hline \multicolumn{4}{|l|}{ inputrelatively } \\
\hline \multicolumn{4}{|l|}{ divided in to groups } \\
\hline $\operatorname{low}$ & control & control & \\
\hline m edium & $-0.056(-0.505,0392) 0.805$ & $-0.156(-0.619,0307)$ & 0500 \\
\hline high & $-0.052(-0.540,0.436) 0.835$ & $-0.172(-0.752,0-409)$ & 0.563 \\
\hline$P$ for trend & 0.849 & & 0571 \\
\hline
\end{tabular}

